\title{
Tax Compliance and Firms' Strategic Interdependence
}

\author{
Ralph Bayer* and Frank **Cowell \\ *University of Adelaide \\ and
}

**London School of Economics

DARP 81

February 2006
The Toyota Centre

Suntory and Toyota International

Centres for Economics and Related

Disciplines

London School of Economics

Houghton Street

London WC2A 2A

$(+44020) 79556674$ 


\begin{abstract}
We focus on a relatively neglected area of the tax-compliance literature in economics, the behaviour of firms. We examine the impact of alternative audit rules on receipts from a tax on profits in the context of strategic inter-dependence of firms. In the market firms may compete in terms of either output or price. The enforcement policy can have an effect on firms' behaviour in two dimensions - their market decisions as well as their compliance behaviour. An appropriate design of the enforcement policy can thus have a "double dividend" by manipulating firms in both dimensions.
\end{abstract}

- JEL: H20, H21

- Keywords: Tax compliance, evasion, oligopoly

- Correspondence: Prof. F. A. Cowell, STICERD, LSE, Houghton St, London, WC2A 2AE. UK.

- email: f.cowell@1se.ac.uk 


\section{Distributional Analysis Research Programme}

The Distributional Analysis Research Programme was established in 1993 with funding from the Economic and Social Research Council. It is located within the Suntory and Toyota International Centres for Economics and Related Disciplines (STICERD) at the London School of Economics and Political Science. The programme is directed by Frank Cowell. The Discussion Paper series is available free of charge. To subscribe to the DARP paper series, or for further information on the work of the Programme, please contact our Research Secretary, Leila Alberici on:

Telephone: UK+20 79556674

Fax: $\quad$ UK+20 79556951

Email: 1.alberici@1se.ac.uk

Web site: $\quad \underline{\text { http://sticerd.lse.ac.uk/DARP }}$

(C) Authors: Ralph Bayer and Frank Cowell.

All rights reserved. Short sections of text, not to exceed two paragraphs, may be quoted without explicit permission provided that full credit, including $\odot$ notice, is given to the source. 


\section{Introduction}

The behaviour of firms is sometimes glossed over in the analysis of economic analysis of tax policy. In the analysis of tax compliance it is often omitted altogether. This omission is rather odd: tax inspectors typically use background knowledge about markets and industries in order to refine the monitoring and auditing process and, even if this knowledge is exercised in rule-of-thumb fashion, one would expect it to be in conformity with rational economic principles. Of course, firms do make an appearance in the standard compliance literature, but only in a rather specialised manner and in connection with rather specialised questions. In this paper we take a step toward a richer analysis by focusing on corporate tax-evasion and market decisions in an oligopolistic setting. We examine the impact of alternative audit rules on receipts from a tax on profits, allowing for both compliance responses and output or price responses by the firms. Why does this alternative focus make such a difference to the analysis?

Most models in the literature focus on a simple proportionate audit rule in an adapted version of the Allingham and Sandmo (1972) model, as though firms habitually play the dual roles of producers and gamblers. In nearly all the standard models of corporate compliance there is a fundamental separation result between the production and concealment activities. This conclusion appears to be robust to alternative assumptions about market structure and the specifications of firms' objectives.

However, taxes are not neutral in a setting where the behaviour of the tax authority depends on all the declarations in a particular market. The tax authority can exploit this market-based information and so, in the light of this, we investigate the implications of using a more intelligent audit rule that is easily implementable. The idea is that a change in audit rule will introduce a regime where tax enforcement can influence output decisions. By conditioning an individual audit on the declaration of all firms the authority creates an externality. The externality can be seen as generating two dividends: (a) less tax evasion (b) an efficiency improvement. The reduction in tax evasion is a direct result of the tax authority's making better use of available information from the collection of firms. The move toward static efficiency arises because of an induced increase in output generated by the switch in enforcement regime.

The paper is organised as follows. Section 2 reviews the story of corporate tax compliance as conventionally presented in the literature and outlines the model presented here; section 3 examines the equilibrium behaviour of

firms in the two main dimensions and sections 4 to 6 present the main res- 
ults, while Section 7 provides an example illustrating the results. Section 8 concludes.

\section{The setting and model outline}

\subsection{Background}

The literature on models of corporate tax compliance usually focuses on one of two relatively simple market structures - competitive price taking or monopoly. The elements of such a model are simple: a risk-neutral pricetaking firm with constant marginal costs and a determinate demand curve faces a proportionate profits tax The sole source of uncertainty is created by a combination of the firm's actions (the firm can conceal profit, but at a cost) and the government's tax audit (a given audit probability with a known penalty proportionate to the amount concealed). The firm conceals up to the point where the marginal cost of concealment equals the marginal reduction of expected tax rate, a rule that is independent of the firm's output level (Cowell 2004).

The advantage of this approach is its simplified behavioural analysis of the tax-evading firm: the "production department" can get on with determining the level of output in the light of market conditions; the "taxmanagement department" separately decides on matters of profit declaration. But there are several three causes for concern:

- The separability result is clearly artificial and it is not clear that it would survive in a more interesting model of the industry.

- The type of audit rule used is naive in that it does not make use of low-cost or costless information that would be available to the taxauthority from the firms' reports.

- The argument that taxation policy has no effect on output seems inappropriate in the light of the perception that corporate taxation does have an effect on firms' activities. Of course this perception may be misplaced, but it would be useful to know whether there is a good theoretical case for considering a real effect of taxation and tax-enforcement policy.

To address these questions we develop a simple model that will permit a somewhat richer version of market structure and behaviour by the tax authority. The model consists of a conventional story of individual firms, an 
industry with a given number of firms, a simple tax function and an audit rule. We will briefly examine each of these in turn.

\subsection{The industry}

We focus on an oligopolistic market with a fixed number of firms producing a single homogeneous output. The firms compete in a standard model of market interaction: we will consider both quantity-competition (Cournot) and price-competition (Bertrand) versions of the model. Each firm has a simple production technology, the details of which are subsumed within a conventional profit function. It faces a requirement to pay tax and knows that it has opportunities for evasion. This enables us to focus on perhaps the most appealing and relatively uncomplicated case of strategic interdependence amongst firms in order to examine the potential role of taxation policy in a market form that is not purely mechanistic. One consequence of this is that, in the context of quantity competition, we would expect the standard Cournot-Nash equilibrium to emerge in which output is above the level corresponding to joint-profit maximisation but below that characterising economic efficiency. In what follows we describe the environment for firms competing in quantities. The case of price competition can be obtained by exchanging $q_{i}$ (quantity produced by firm $i$ ) for $p_{i}$ (price set by firm $i$ ) as arguments of the gross profit functions.

\subsection{Taxation and profits}

Let us set out the role of the tax system in the objective function for the firms. Formally there is a population $N:=\{1, \ldots, n\}$ of firms where $N$ is exogenous. Firm $i$ makes gross profit $\pi_{i}^{g}(\mathbf{q})$ where

$$
\mathbf{q}:=\left(q_{1}, q_{2}, \ldots, q_{i}, \ldots q_{n}\right)
$$

is the vector of quantities produced. Each firm has to declare profits. The declaration of firm $i$ is denoted by $d_{i}$ and the vector of declarations is written

$$
\mathbf{d}:=\left(d_{1}, d_{2}, \ldots, d_{i}, \ldots, d_{n}\right) .
$$

Rather than limiting itself to treating each $d_{i}$ in isolation the tax authority can base an audit rule on the information provided by the collection of declarations $\mathbf{d}$. This crucial point is developed in section 2.6. 
We assume a linear profits tax and a fine for detected tax evasion, that is proportional to the concealed profit. ${ }^{1}$ There is no loss-offset or compensation: subsidies are not given for losses, nor are bonuses paid for revealed over-compliance. So the legal tax liability is defined as

$$
T_{i}^{l e g}=t \max \left[0, \pi_{i}^{g}(\mathbf{q})\right]
$$

where $t$ is the tax rate. The tax payable depending on the profit declaration if no audit takes place is

$$
T_{i}^{\text {pay }}=\max \left[0, t d_{i}\right] .
$$

So the profit net of taxes if no audit takes place can be written as:

$$
\bar{\pi}_{i}\left(d_{i}, \mathbf{q}\right):=\left\{\begin{array}{cc}
\pi_{i}^{g}(\mathbf{q})-t d_{i} & \text { if } d_{i} \geq 0 \\
\pi_{i}^{g}(\mathbf{q}) & \text { otherwise }
\end{array}\right.
$$

After an audit, if a firm is found to have underpaid tax it is required to make up the shortfall and also to pay a fine $F$ :

$$
\underline{\pi}_{i}\left(d_{i}, \mathbf{q}\right):=\pi_{i}^{g}(\mathbf{q})-t \max \left[0, \pi_{i}^{g}(\mathbf{q})\right]-F\left(\pi_{i}^{g}(\mathbf{q}), d_{i}\right)
$$

We assume a fine proportional to undeclared profit. With the proportionality factor $f$ the fine becomes:

$$
F\left(\pi_{i}^{g}(\mathbf{q}), d_{i}\right):=\left\{\begin{array}{cc}
f\left[\pi_{i}^{g}(\mathbf{q})-d_{i}\right] & \text { if } d_{i}<\pi_{i}^{g}(\mathbf{q}) \\
0 & \text { otherwise }
\end{array}\right.
$$

\subsection{Concealment cost}

Effective concealment requires that the firm incur a real resource cost. ${ }^{2}$ We do not assume a specific functional form for this. However, it seems reasonable to assume that the concealment cost rises with the profit concealed. Additionally, we assume that the marginal concealment cost are also increasing in the profit concealed. For declarations which are not higher than the actual profit $\left(d_{i} \leq \pi_{i}^{g}\right)$ we have

$$
\begin{aligned}
C\left(\pi_{i}^{g}-d_{i}\right) & \geq 0 \\
C^{\prime} & >0 \\
C^{\prime \prime} & >0 .
\end{aligned}
$$

\footnotetext{
${ }^{1}$ Note that the way the fine is defined may play a crucial role for the effect changes in the tax rate have on declaration behaviour. For the purposes of our paper, which focuses on the analysis of the impact of a relative audit rule, the formulation of the fine is not crucial.

${ }^{2}$ These cost e.g. could stem from buying in specialist advise, reorganizing transaction paterns or purchasing avoidance schemes.
} 
where primes denote derivatives. We further assume that there are no benefits for over-reporting and that concealment cost are zero for truthful declarations:

$$
\begin{aligned}
C\left(\pi_{i}^{g}-d_{i}\right) & =0 \text { for } d_{i}>\pi_{i}^{g}, \\
C(0) & =0 .
\end{aligned}
$$

\subsection{Objective function}

For expositional reasons, we assume the managers of the firm to be riskneutral. Our analysis does not crucially depend on the risk preferences assumed. ${ }^{3}$ Let us denote the audit probability for firm $i$, which is determined by the tax authority, as $\beta(i, \mathbf{d}) .{ }^{4}$ The audit probability for firm $i$ may or may not depend on the vector of all declarations in the industry. If it does depend on $\mathbf{d}$ in a certain way we will speak of a relative audit rule (see section 2.6 for details). If the audit probability is fixed and does not depend on either the declaration of the firm in question or the declarations of the other firms in the industry then we speak of a fixed audit rule.

Given a relative audit rule we can write the objective function of the firm as its expected payoff:

$$
\mathbf{E}\left(\pi_{i}(\mathbf{d}, \mathbf{q})\right):=\beta(i, \mathbf{d}) \underline{\pi}_{i}\left(d_{i}, \mathbf{q}\right)+[1-\beta(i, \mathbf{d})] \bar{\pi}_{i}\left(d_{i}, \mathbf{q}\right)-C\left(\pi_{i}^{g}(\mathbf{q}), d_{i}\right)
$$

The appropriate objective function under the fixed audit rule can be found by replacing the expression $\beta(i, \mathbf{d})$ by a given number $\beta^{f i x} \in(0,1)$ in Equation (3).

\subsection{Audit rule}

Suppose the authority assigns the audit probability $\beta(i, \mathbf{d})$ to firm $i$ where the individual audit probability is conditional on $\mathbf{d}$. Then we can use the following definition:

\footnotetext{
${ }^{3}$ In contrast to a large body of literature we does not analyze the impact of tax rates on evasion where risk preferences and fine functions are crucial for the results.

${ }^{4}$ In what follows we will use the term audit probability. This implies that the authority conducts randum full-scale audits, which perfectly reveal evasion. However, in reality authorities audit large firms every year. Due to the complexity these audits are only partial and do not reveal the true profit with certainty. Under such a scenario $\beta_{i}$ can be seen as the detection probability, which can be increased by the authority by increasing the audit effort.
} 
Definition $1 A$ relative audit rule is a function $\beta: N \times R^{n} \mapsto[0,1]$ that satisfies the following conditions:

$$
\begin{aligned}
& \frac{\partial \beta(i, \mathbf{d})}{\partial d_{i}} \leq 0 \quad \forall i \in\{1,2, \ldots, n\} \\
& \frac{\partial \beta(i, \mathbf{d})}{\partial d_{j}} \geq 0 \quad \forall j \neq i \in\{1,2, \ldots, n\} \\
& \sum_{i=1}^{n} \frac{\partial \beta(i, \mathbf{d})}{\partial d_{j}}=0 \quad \forall j \in\{1,2, \ldots, n\}
\end{aligned}
$$

The first property (D1) takes into account that ceteris paribus a higher profit declaration should lead to a lower audit probability. Lower declarations make the authority more suspicious and induce higher audit probabilities. Condition D2 captures the relative nature of the audit rule: if a competitor increases its declaration then this increases $i$ 's probability of being audited. The rationale behind this property is that the observation of a high declaration of one firm makes the authority believe that the profit situation in the industry was good for the fiscal year in question. Then it should shift its attention to the firms with comparatively low declarations, such as $i$, because it becomes more likely that those firms have under-reported their profits.

Put differently, an authority that does not know the profit situation in an industry on the one hand and that on the other hand believes that profits in an industry are correlated, should put a higher probability of tax evasion on a received declaration if the declarations of other firms increase. This strategy corresponds to an implicit model of industry profits that contains an industry-specific common shock component the authority cannot observe. However, observing the level of declarations the authority can draw inferences on this common shock component.

So differences in declarations indicate firm-specific unobserved shocks and/or tax evasion. A higher profit declaration of one firm renders tax evasion of the others more likely for their given declarations.

The third part of the definition (D3) captures the widely applied practice of tax authorities to assign a certain amount of resources to an industry. ${ }^{5}$ Technically (D3) keeps the expected number of audits in an industry constant.

\footnotetext{
${ }^{5}$ This is done implicitly in countries like the United States or Germany while in other countries (e.g. Australia) the targeted industries are made public.
} 


\section{Equilibrium}

In this section we outline the optimisation problem of the firms. We focus on quantity competition under a relative audit rule. Bertrand competition is obtained by changing the choice variable in the competition stage. For a fixed audit rule we just set the partial derivatives of the detection probability with respect to the declaration of any firm to zero.

\subsection{Information and timing}

Let us focus on quantity competition in the first instance. We can imagine the following simple sequence of decisions and actions.

0 Firms learn the tax and fine system and the audit rule that is in place for the coming tax year.

1 Firms choose quantities.

2 Firms observe the gross profits of all market participants and then choose their profit declarations.

It is the interaction of firms in stages one and two that makes the problem particularly interesting. Implicitly, we assume that the firms have an information advantage over the authority. Firms are better informed about the level of profits within an industry than the tax authority; this gives the relative audit rule "bite." This situation could be applicable in an industry with stochastic demand shocks, for example: a firm can infer from its own profit what the equilibrium profits of the other firms must look like. In standard models the extreme assumption is made that firms have no information advantage over the tax authority at all; for simplicity we take the opposite extreme where firms know the profits in the industry, while the authority does not. ${ }^{6}$

\subsection{The declaration stage}

We begin with the stage where firms make the declaration to the tax authority. The firms will treat the gross profits as given when they decide about their declarations. First of all, note that there is no incentive for a firm

\footnotetext{
${ }^{6}$ An intermediate model where firms obtain a more accurate signal about the level of profits in the industry than the tax authority seems to be most realistic. We chose the extreme case model for two reasons: (a) tractability and (b) the basic intuition of an externality imposed by a relative audit rule is not obscured by the signal sturcture.
} 
to declare more than the profit actually made, since no reward is given for over-compliance. Additionally, it is clear that the optimal declaration for a firm which incurs losses is to report truthfully. This is the case, because declaring a higher loss than actually suffered does not lead to any subsidies, but concealment cost and potentially fines have to be paid. We now turn to the situation where tax evasion is optimal. Assume a positive gross profit $\left(\pi_{i}^{g}>0\right)$. Then the first-order condition for an interior solution is given by:

$$
\frac{\partial E \pi_{i}}{\partial d_{i}}:=\frac{\partial \beta(i, \mathbf{d})}{\partial d_{i}}\left[\underline{\pi}_{i}-\bar{\pi}_{i}\right]+\beta\left[\frac{\partial \underline{\pi}_{i}}{\partial d_{i}}-\frac{\partial \bar{\pi}_{i}}{\partial d_{i}}\right]+\frac{\partial \bar{\pi}_{i}}{\partial d_{i}}-\frac{\partial C_{i}}{\partial d_{i}}=0
$$

The second-order condition is:

$$
\frac{\partial^{2} E \pi_{i}}{\partial d_{i}^{2}}:=\frac{\partial^{2} \beta(i, \mathbf{d})}{\partial d_{i}^{2}}\left[\underline{\pi}_{i}-\bar{\pi}_{i}\right]+2 \frac{\partial \beta(i, \mathbf{d})}{\partial d_{i}}\left[\frac{\partial \underline{\pi}_{i}}{\partial d_{i}}-\frac{\partial \bar{\pi}_{i}}{\partial d_{i}}\right]-\frac{\partial^{2} C_{i}}{\partial d_{i}^{2}}<0
$$

Note that $\underline{\pi}_{i}-\bar{\pi}_{i} \leq 0, \partial \beta(i, \mathbf{d}) / \partial d_{i}<0, \partial \underline{\pi}_{i} / \partial d_{i}-\partial \bar{\pi}_{i} / \partial d_{i}=f+t>0$, and $\partial^{2} C_{i} / \partial d_{i}^{2}>0$. Therefore a sufficient condition for the second-order condition to be satisfied is

$$
\frac{\partial^{2} \beta(i, \mathbf{d})}{\partial d_{i}^{2}} \leq 0
$$

To simplify matters we only consider audit rules that satisfy (D4).

Given positive profits and the global concavity of the objective function in firm $i$ 's own declaration we can establish conditions for an interior solution. There is an interior solution whenever

$$
\left.\frac{\partial E \pi_{i}}{\partial d_{i}}\right|_{d_{i}=0}>0>\lim _{d_{i} \rightarrow \pi_{i}^{g}} \frac{\partial E \pi_{i}}{\partial d_{i}}
$$

Inspection shows that an interior solution is likely whenever

a. marginal concealment costs are high for extensive tax evasion, which prevents zero-declarations, and

b. a generally low level of detection probabilities and a low marginal evasion-cost for the first unit of profit concealed exist, which gives incentives for evasion.

However, because of the endogeneity of the detection probability, there is no general condition which depends only on parameters that will ensure an internal solution. 


\subsection{The market-response stage}

Now consider the stage that determines the firms' profits: depending on the assumptions made about the nature of competition in the market each firm makes a decision about output or price. Clearly the standard issue of the existence of an equilibrium in pure strategies will arise. Assuming that the strategy space is compact and non-empty and that the objective functions are continuous and strictly quasi-concave in the choice variable ensures a unique equilibrium in pure strategies - see Fudenberg and Tirole (1991), p.34. To avoid complications we assume for now that these conditions to hold. ${ }^{7}$ The assumption of uniqueness is dropped in the next section.

Take a market where firms compete in quantities - the case of competition on price is analysed below. Then the first-order condition for optimal output given that firms will file (and expect the competitors to file) an optimal tax return for any possible profit distribution can be written as:

$$
\frac{\partial E \pi_{i}}{\partial q_{i}}:=\frac{\partial \beta(i, \mathbf{d})}{\partial q_{i}}\left[\underline{\pi}_{i}-\bar{\pi}_{i}\right]+\beta^{*}(i, \mathbf{d})\left[\frac{\partial \underline{\pi}_{i}^{*}}{\partial q_{i}}-\frac{\partial \bar{\pi}_{i}^{*}}{\partial q_{i}}\right]+\frac{\partial \bar{\pi}_{i}^{*}}{\partial q_{i}}-\frac{\partial C_{i}^{*}}{\partial q_{i}}=0
$$

The asterisks indicate that the changes of the optimal declaration (of all firms) due to change in the quantity supplied have to be taken into account. Note that the change in the equilibrium audit-probability $\partial \beta^{*}(i, \mathbf{d}) / \partial q_{i}$ includes the effects of declaration changes of all firms due to the change in observed profits. Solving the first-order condition for the declaration (4) for $C^{\prime}$ and substituting in the first-order condition from above gives:

$$
\sum_{j \neq 1}^{n} \frac{\partial \beta(i, \mathbf{d})}{\partial d_{j}^{*}} \frac{\partial d_{j}^{*}}{\partial q_{i}}\left[\underline{\pi}_{i}-\bar{\pi}_{i}\right]+\frac{\partial \pi_{i}^{g}}{\partial q_{i}}\left[1-t+t \beta^{*}(i, \mathbf{d})+\left[\underline{\pi}_{i}-\bar{\pi}_{i}\right] \frac{\partial \beta(i, \mathbf{d})}{\partial d_{i}^{*}}\right]=0
$$

The set of first-order conditions, one for each firm, characterises the equilibrium. Using this characterisation of equilibrium we can now present the main results in three steps: sections 4 to 6 .

\section{Compliance decisions}

In this section we compare the different effects fixed and relative audit rules have on the extent of tax evasion. We find that a relative audit rule leads to

\footnotetext{
${ }^{7}$ A compact strategy set results from restricting the feasible quantities to $q_{i} \in\left[0, q^{\max }\right]$ $\forall i$, where $q^{\max }$ is the biggest production quantity that is physically feasible. The objective functions are obviously continuous. Quasi-concavity can be achieved by choosing appropriate demand, cost and detection- probability functions.
} 
less tax evasion than a comparable fixed audit rule if the declaration stage has an interior solution. The result derived is not dependent on whether firms compete in quantities or prices.

The intuition for the result is rather simple: in addition to the typical incentives provided by a fixed detection probability and the corresponding fine, a relative audit rule provides a further incentive to increase the declaration, as this decreases the detection probability. Suppose we have a fixed detection probability $\beta^{f i x}$ then the first-order condition of an individual firm in the declaration stage becomes:

$$
\frac{\partial E \pi_{i}^{f i x}}{\partial d_{i}}:=\beta^{f i x}\left[\frac{\partial \underline{\pi}_{i}}{\partial d_{i}}-\frac{\partial \bar{\pi}_{i}}{\partial d_{i}}\right]+\frac{\partial \bar{\pi}_{i}}{\partial d_{i}}-\frac{\partial C_{i}}{\partial d_{i}}=0
$$

Recall that the first-order condition under a relative audit rule is given by

$$
\frac{\partial E \pi_{i}}{\partial d_{i}}:=\frac{\partial \beta(i, \mathbf{d})}{\partial d_{i}}\left[\underline{\pi}_{i}-\bar{\pi}_{i}\right]+\beta\left[\frac{\partial \underline{\pi}_{i}}{\partial d_{i}}-\frac{\partial \bar{\pi}_{i}}{\partial d_{i}}\right]+\frac{\partial \bar{\pi}_{i}}{\partial d_{i}}-\frac{\partial C_{i}}{\partial d_{i}}=0 .
$$

In order to compare the two rules we need a criterion that makes the two regimes comparable. Hence we require the equilibrium detection probability in the relative-rule scenario to be equal to the fixed detection probability. This ensures that the audit costs incurred by the authority are equal under both regimes. Setting $\beta=\beta^{\text {fix }}$ and keeping the profits the same in both cases implies that the two first-order conditions only differ by the term

$$
\frac{\partial \beta(i, \mathbf{d})}{\partial d_{i}}\left[\underline{\pi}_{i}-\bar{\pi}_{i}\right]
$$

This is just the additional incentive to increase the declaration in order to reduce the detection probability, which leads to higher declarations under a relative audit rule.

Proposition 1 In an interior declaration equilibrium a relative audit rule leads to less evasion than a fixed rule with the same detection probability.

Proof. Pick an interior $d_{i} \in\left(0, \Pi_{i}\right)$ such that for a given $\beta=\beta^{\text {fix }}$ condition (7) holds. Then under the fixed audit rule the declaration is optimal. For this $d_{i}$ the marginal expected profit of declaring an additional dollar under a fixed rule is

$$
\frac{\partial \beta(i, \mathbf{d})}{\partial d_{i}}\left[\underline{\pi}_{i}-\bar{\pi}_{i}\right]
$$


which is positive for an interior solution as $\partial \beta(i, \mathbf{d}) / \partial d_{i}<0, \underline{\pi}_{i}-\bar{\pi}_{i}<0$. Assumption (D4) ensures global concavity of $E \pi_{i}$ in $d_{i}$, which implies the claimed property.

So, in a situation where there is competition among the few, switching to a more intelligent audit rule yields an immediate dividend - less evasion.

\section{Output decisions}

However, there is more that can be said on behalf of the relative audit rule. As we noted earlier (section 2.1), in simple competitive and noncompetitive models the tax-enforcement parameters do not distort output although this result depends on the way in which the audit probability and penalty rate are formulated (Lee 1998). So too in our model: it is clear that there is no effect on output if audit probabilities are independent of the declarations.

In what follows we will show that for typical Cournot games with unique equilibria in pure strategies a switch to relative auditing will increase aggregate quantity and thus efficiency. We exclude underlying Cournot games with multiple equilibria in order to have a definite reference for our comparative statics analysis. ${ }^{8}$ Suppose we have a smooth Cournot oligopoly with a compact strategy space where inverse demand $p(Q)$ is decreasing and logconcave in $Q$. Furthermore assume that production costs $K$ are such that $K_{i}^{\prime \prime}\left(q_{i}\right)-p^{\prime}(Q)>0$ for all firms. Note that this does not exclude asymmetry with regard to the cost functions. The conditions above are well-known to be sufficient for N-player Cournot games to have a unique Nash equilibrium in pure strategies. ${ }^{9}$ In what follows we refer to oligopolies as regular if they satisfy these conditions. In a such regular oligopolies the best response functions of firms have non-positive slopes larger than -1 :

$$
\frac{d q_{i}^{*}}{d Q_{-i}}=-\frac{\partial^{2} \Pi_{i}}{\partial q_{i} \partial Q_{-i}} / \frac{\partial^{2} \Pi_{i}}{\partial q_{i}^{2}}=-\frac{p^{\prime}+p^{\prime \prime} q_{i}}{2 p^{\prime}+p^{\prime \prime} q_{i}-K_{i}^{\prime \prime}}>-1 .
$$

We first establish that the best-response quantity of an individual firm under relative auditing is larger than under a fixed audit rule. As mentioned above under a fixed audit rule the quantity decision is equivalent to the decision in an oligopoly without taxation and tax enforcement.

\footnotetext{
${ }^{8}$ Note that we do not exclude games where the resulting Cournot game with relative auditing has multiple equilibria (potentially in mixed strategies) as long as the underlying Cournot game has a unique equilibrium in pure strategies.

${ }^{9}$ See Vives (1999), Theorems 2.7 and 2.8 .
} 
Proposition 2 Under an independent audit rule output is independent of the evasion decision and equals the Cournot quantities.

Proof. In the declaration stage the first-order condition for an independent audit rule is identical to the one under a relative audit rule from (4). The only difference is that the first-order conditions of different firms are not linked through $\beta$ and $\partial \beta(i, \mathbf{d}) / \partial d_{i}^{*}$. The first-order condition on the output stage is also very similar to the corresponding condition under a relative audit rule (5). The only difference here is that $\partial \beta\left(i, d_{i}\right) / \partial q_{i}$ does not contain any indirect influences via changes in the competitors declarations due to a changed output. ${ }^{10}$ Substituting the first-order condition for declaration into the first-order condition for output choices gives the condition

$$
\frac{\partial \pi_{i}^{g}}{\partial q_{i}}\left[1-t+t \beta^{*}\left(i, d_{i}\right)+\left[\underline{\pi}_{i}-\bar{\pi}_{i}\right] \frac{\partial \beta\left(i, d_{i}\right)}{\partial d_{i}^{*}}\right]=0
$$

which only holds if $\partial \pi_{i}^{g} / \partial q_{i}=0$. This implies that the oligopolists choose the Cournot quantity.

We now turn to relative auditing. Denote the best response of firm $i$ in the regular Cournot game as $B R_{i}^{C}\left(\mathbf{q}_{-i}\right)$, while $B R_{i}^{R}\left(\mathbf{q}_{-i}\right)$ gives the best response correspondence under a relative audit rule.

Lemma 3 Assume that the market organisation has the form of a regular Cournot oligopoly. Then

$$
B R_{i}^{R}\left(\mathbf{q}_{-i}\right)>B R_{i}^{C}\left(\mathbf{q}_{-i}\right)
$$

wherever $B R_{i}^{C}\left(\mathbf{q}_{-i}\right)>0$.

Proof. Take the first-order condition for the quantity choices from (6):

$$
\underbrace{\sum_{j \neq i}^{n} \frac{\partial \beta^{*}(i, \mathbf{d})}{\partial d_{j}^{*}} \frac{\partial d_{j}^{*}}{\partial q_{i}}\left[\underline{\pi}_{i}-\bar{\pi}_{i}\right]}_{(a)}+\underbrace{\frac{\partial \pi_{i}^{g}}{\partial q_{i}}}_{(b)} \underbrace{\left[1-t+t \beta^{*}(i, \mathbf{d})+\left[\underline{\pi}_{i}-\bar{\pi}_{i}\right] \frac{\partial \beta^{*}(i, \mathbf{d})}{\partial d_{i}^{*}}\right]}_{(c)}=0
$$

Note that term (c) is positive, since $1-t+t \beta^{*}(i, \mathbf{d})>0, \underline{\pi}_{i}-\bar{\pi}_{i} \leq 0$, and $\partial \beta^{*}(i, \mathbf{d}) / \partial d_{i}^{*} \leq 0$. So the sign for (b) has to be the opposite of term (a); and if (a) is zero then (b) has to be zero as well. Recall that $\partial \pi_{i}^{g} / \partial q_{i}=0$ is the first-order condition for optimal output in a Cournot oligopoly. So for

\footnotetext{
${ }^{10}$ For the totally fixed audit rule analysed above this derivative vanishes entirely.
} 
a higher output, which is closer to the socially efficient output $\partial \pi_{i}^{g} / \partial q_{i}<0$ has to hold. This implies that (a) needs to be positive for our claim to be true. As $\underline{\pi}_{i}-\bar{\pi}_{i}<0$ and $\partial \beta^{*}(i, \mathbf{d}) / \partial d_{j}^{*}>0$ for any interior solution, the crucial question is whether $\partial d_{j}^{*} / \partial q_{i}<0$ holds. The declaration of firm $j$ will be influenced by a change of $q_{i}$ through two different channels: the change in the own profit and the reactions of other firms due to their changed profits. Using the implicit function theorem we can write firm $j$ 's subgame perfect reaction to firm $i$ changing its quantity as:

$$
\frac{\partial d_{j}^{*}}{\partial \pi_{j}^{g}} \frac{\partial \pi_{j}^{g}}{\partial q_{i}}=-\frac{1}{\Delta}\left[C^{\prime \prime}-(f+t) \frac{\partial \beta^{*}(j, \mathbf{d})}{\partial d j}\right] \frac{\partial \pi_{j}^{g}}{\partial q_{i}}
$$

where

$$
\Delta:=\frac{\partial^{2} E \pi_{j}}{\partial d_{j}^{2}} .
$$

The whole term is negative if $\Delta<0, C^{\prime \prime}>0, \partial \beta(j, \mathbf{d}) / \partial d_{j}<0$, and $\partial \pi_{j}^{g} / \partial q_{i}<0$. If so, then (a) is negative, as all competitors have qualitatively identical first-order effects. This shows that the optimal quantity (or all optimal quantities if $B R_{i}^{R}\left(\mathbf{q}_{-i}\right)$ is a correspondence rather than a function) is higher than the best response under Cournot for the same output vector of the other firms:

$$
B R_{i}^{R}\left(\mathbf{q}_{-i}\right)>B R_{i}^{C}\left(\mathbf{q}_{-i}\right) \forall i, \mathbf{q}_{-i}
$$

This result is sufficient to ensure that the aggregate quantity under a relative auditing rule is larger if a) the oligopoly is regular and symmetric and b) the equilibrium under relative auditing is also symmetric. However, by using some properties of a regular oligopoly we can generalise this result considerably. Denote the aggregate equilibrium quantities under a relative rule as $Q_{R}^{*}$ and under ordinary Cournot (which is the same as under a fixed rule) as $Q_{C}^{*}$. Then we can establish the following more general result.

Proposition 4 If the underlying Cournot oligopoly is regular then any interior equilibrium outcome under a relative auditing rules satisfies $Q_{R}^{*}>Q_{C}^{*}$.

Proof. Fix the quantities of $N-2$ firms and investigate the location of the best responses of the two remaining firms, say 1 and 2, under relative auditing. Inequality (10) from the previous lemma tells us that for every given quantity vector of the $N-2$ remaining firms

$$
\begin{aligned}
& B R_{1}^{R}\left(q_{2}\right)>B R_{1}^{C}\left(q_{2}\right) \\
& B R_{2}^{R}\left(q_{1}\right)>B R_{2}^{C}\left(q_{1}\right)
\end{aligned}
$$


hold. So the candidates $q_{1}$ and $q_{2}$ for mutual best responses given the others' quantities have to jointly satisfy:

$$
q_{1}>B R_{1}^{C}\left(q_{2}\right)
$$

and

$$
q_{2}>B R_{2}^{C}\left(q_{1}\right)
$$

As $B R_{2}^{C}\left(q_{1}\right)$ is strictly monotonous in a regular oligopoly for interior equilibrium we can invert the second condition and write:

$$
\begin{aligned}
& q_{1}>B R_{1}^{C}\left(q_{2}\right) \vee q_{1}>B R_{2}^{-1}\left(q_{2}\right), \text { or } \\
& q_{1}>\max \left\{B R_{1}^{C}\left(q_{2}\right), B R_{2}^{-1}\left(q_{2}\right)\right\}
\end{aligned}
$$

where $B R_{2}^{-1}\left(q_{2}\right)$ denotes the inverse best response function of firm 2. From condition (8) for a regular Cournot game we can conclude that:

$$
\begin{aligned}
\frac{d}{d q_{2}} B R_{1}^{C}\left(q_{2}\right) & >-1 \forall q_{2} \\
\frac{d}{d q_{2}} B R_{2}^{-1}\left(q_{2}\right) & <-1 \forall q_{2}
\end{aligned}
$$

We have $B R_{1}^{C}\left(q_{2}^{*}\right)=B R_{2}^{-1}\left(q_{2}^{*}\right)$, where $q_{2}^{*}$ is firm two's quantity in the equilibrium of the reduced two-firm game for given quantities of the others. Because the slopes are different this implies:

$$
q_{1}>\left\{\begin{array}{ccc}
B R_{2}^{-1}\left(q_{2}\right) & \text { for } \quad q_{2}<q_{2}^{*} \\
B R_{1}^{C}\left(q_{2}\right) & \text { for } \quad q_{2} \geq q_{2}^{*}
\end{array}\right.
$$

Now given that the isoquant for aggregate output $\bar{q}_{1}\left(q_{2}\right)$ for the Cournot equilibrium of the reduced game has slope -1 and satisfies $\bar{q}_{1}\left(q_{2}^{*}\right)=B R_{1}^{C}\left(q_{2}^{*}\right)=$ $B R_{2}^{-1}\left(q_{2}^{*}\right)$ we can conclude:

$$
q_{1}>\bar{q}_{1}\left(q_{2}\right) \forall q_{2}
$$

So the aggregate quantity of two firms under relative auditing is larger than without for any given quantity of the others. As this is true for any two firms and any given quantity of the other firms we can conclude that any equilibrium output vector $\mathbf{q}_{R}^{*}$ is such that $Q_{R}^{*}>Q_{C}^{*}$.

The result, illustrated in Figure 1, might seem surprising. After all, what does a firm gain from extending its output beyond the Cournot level? By increasing output in this way it would seem that a firm reduces its own gross 


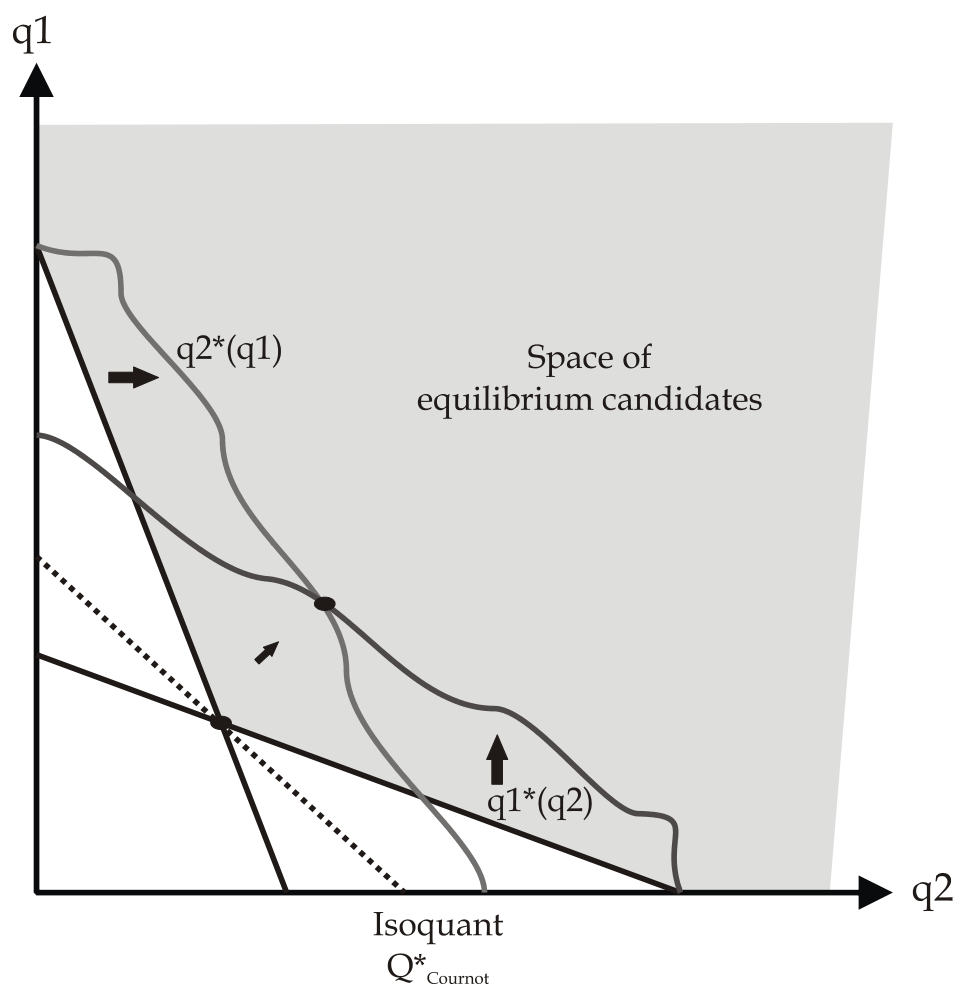

Figure 1: Cournot equilibrium under relative auditing

profit and the gross profits of the competitors; this would seem to be a loss rather than a gain.

However, recall the role of the informational externality here. As $q_{i}$ increases, the profits of other firms $\pi_{-i}^{g}$ fall, so $d_{-i}^{*}$, the optimal declarations of the other firms, fall. Therefore the probability of audit of firm $i$ decreases, which in turn gives firm $i$ some more scope for evasion. By increasing output beyond the Cournot quantity a firm intends to trade some gross profit for a better environment for evasion. This externality can easily be identified by the differences in the first-order conditions for the quantity choices under the different rules. Comparing the first-order conditions shows that the externality under relative auditing is given by the term

$$
\sum_{j \neq i}^{n} \frac{\partial \beta^{*}(i, \mathbf{d})}{\partial d_{j}^{*}} \frac{\partial d_{j}^{*}}{\partial q_{i}}\left[\underline{\pi}_{i}-\bar{\pi}_{i}\right],
$$


which describes the influence of firm $i$ 's quantity on $i^{\prime}$ s expected payoff by the indirect effect on the other firms' declarations and hence firm i's audit probability. As the other firms will decrease their declaration if their profit is decreased, which decreases firm $i$ 's audit probability, firm $i$ has an incentive to sabotage the other firms' profits by producing more than under the equilibrium of the underlying Cournot oligopoly.

So increasing output as described above reduces the impact of the externality imposed by the authority's relative audit rule. A lower gross profit helps closing the gap of the net profits between the situation after an audit $(\underline{\pi})$ and the situation where no audit took place $(\bar{\pi})$. Since all firms have the same incentives this does not really work for the firms, they all try to reduce the impact of the externalities by increasing their quantities. So they are all worse off than if they just had produced the Cournot quantity on the first stage. The externalities imposed by the authority on the profit declaration spill over into the output-decision stage.

\section{Price decisions}

Now consider the impact of relative auditing if firms compete in prices. Obviously, a profit-tax enforcement in a deterministic world only makes sense if firms make positive profits. Therefore we consider Bertrand oligopolies with differentiated products only. In what follows we concentrate on an underlying smooth and (strictly) supermodular Bertrand oligopoly with differentiated substitutes, which has a unique equilibrium in pure strategies. A sufficient condition for uniqueness is that the best responses of the firms $\left(B R_{1}(\cdot), \ldots, B R_{N}(\cdot)\right)$ are a contraction, which is satisfied for a range of standard demand and cost systems (Vives 1999, p 150). We will refer to such a price game as a regular Bertrand oligopoly with substitutes.

The second stage of the tax-evasion game is obviously not affected by changing the market structure and choice variable. Firms still maximize their expected payoffs by simultaneously choosing profit declarations given the profits determined in the competition stage. The first-order conditions (4) for optimal declarations do not change. This implies that the first dividend of relative auditing - less evasion for a given auditing budget - is also present under Bertrand competition. The more interesting question is whether a relative audit rule enhances efficiency under Bertrand competition in the same way as it does under Cournot competition. Note that an audit rule enhances efficiency in price competition with differentiated products if it lowers equilibrium prices. Denote the best response of firm $i$ to the 
prices of the competitors in the regular Bertrand game as $B R_{i}^{B}\left(\mathbf{p}_{-i}\right)$, while $B R_{i}^{R}\left(\mathbf{p}_{-i}\right)$ gives the best response correspondence under a relative audit rule.

Lemma 5 Assume that the market organisation has the form of a regular Bertrand oligopoly. Then

$$
B R_{i}^{R}\left(\mathbf{p}_{-i}\right)<B R_{i}^{B}\left(\mathbf{p}_{-i}\right)
$$

wherever $B R_{i}^{B}\left(\mathbf{p}_{-i}\right)>0$

Proof. Take the first-order condition for the price choices, which is (6) with quantities are replaced by the prices:

$$
\underbrace{\sum_{j \neq i}^{n} \frac{\partial \beta^{*}(i, \mathbf{d})}{\partial d_{j}^{*}} \frac{\partial d_{j}^{*}}{\partial p_{i}}\left[\underline{\pi}_{i}-\bar{\pi}_{i}\right]}_{(a)}+\underbrace{\frac{\partial \pi_{i}^{g}}{\partial p_{i}}}_{(b)} \underbrace{\left[1-t+t \beta^{*}(i, \mathbf{d})+\left[\underline{\pi}_{i}-\bar{\pi}_{i}\right] \frac{\partial \beta^{*}(i, \mathbf{d})}{\left.\partial d_{i}^{*}\right]}\right.}_{(c)}=0
$$

To prove our claim we have to show that this first-order condition requires $\partial \pi_{i}^{g} / \partial p_{i}>0$. For this to hold we need (a) to be negative for $p_{i}>0$, as (c) is always positive. As $\underline{\pi}_{i}-\bar{\pi}_{i}<0$ and $\partial \beta^{*}(i, \mathbf{d}) / \partial d_{j}^{*}>0$ for any interior solution, the crucial question is whether $\partial d_{j}^{*} / \partial p_{i}>0$ holds. Using the implicit function theorem we can write firm $j$ 's subgame perfect reaction to firm $i$ changing its price as:

$$
\frac{\partial d_{j}^{*}}{\partial \pi_{j}^{g}} \frac{\partial \pi_{j}^{g}}{\partial p_{i}}=-\frac{1}{\Delta}\left[C^{\prime \prime}-(f+t) \frac{\partial \beta^{*}(j, \mathbf{d})}{\partial d_{j}}\right] \frac{\partial \pi_{j}^{g}}{\partial p_{i}}
$$

where $\Delta$ is again given by (9). Given $\Delta<0, C^{\prime \prime}>0, \partial \beta(j, \mathbf{d}) / \partial d_{i}<0$, and $\partial \pi_{j}^{g} / \partial p_{i}>0$ this term is positive since. Consequently, we need $\partial d_{j}^{*} / \partial p_{i}>0$ for the first-order condition to hold, which proves our claim that

$$
B R_{i}^{R}\left(\mathbf{p}_{-i}\right)<B R_{i}^{B}\left(\mathbf{p}_{-i}\right) \forall i, \mathbf{p}_{-i}>0
$$

With this result in hand it is straightforward to show that a relative audit rule leads to an equilibrium with lower prices for all firms in a regular Bertrand oligopoly with differentiated substitutes.

Proposition 6 If the underlying Bertrand oligopoly with differentiated substitutes is regular then any interior equilibrium outcome under a relative audit rule satisfies $p_{i}^{R}<p_{i}^{B} \forall i$. 
Proof. Fix the prices of $N-2$ firms. According to the lemma above, under relative auditing potential mutual best responses of the two remaining firms 1,2 have to satisfy:

$$
p_{1}<B R_{1}^{B}\left(p_{2}\right)
$$

and

$$
p_{2}<B R_{2}^{B}\left(p_{1}\right) \text {. }
$$

where the fixed prices of the other firms are omitted for simplicity of notation. We can invert the second part of the condition above as best responses are strictly increasing for interior equilibria. The condition for a possible mutual best response becomes:

$$
p_{1}<\max \left\{B R_{1}^{B}\left(p_{2}\right), B R_{2}^{-1}\left(p_{2}\right)\right\} .
$$

As both $B R_{1}^{B}\left(p_{2}\right)$ and $B R_{2}^{-1}\left(p_{2}\right)$ are increasing in $p_{2}$ with a fixed point $\left(p_{1}^{B}, p_{2}^{B}\right)$, which is the Bertrand equilibrium in the reduced game of firms 1 and 2 for given prices of the other firms, we can conclude that $p_{1}^{R}<p_{1}^{B}$. Using the same logic we can show that $p_{2}^{R}<p_{2}^{B}$. This result holds for any two firms and any price vector of the other firms. Therefore we can conclude that in equilibrium $p_{i}^{R}<p_{i}^{B} \forall i$.

Figure 2 illustrates the logic of the proof above. Note that if the underlying Bertrand oligopoly is supermodular, but with multiple equilibria, then there always exists an equilibrium under a relative auditing rule with prices lower than the lowest equilibrium prices under a fixed rule. ${ }^{11}$

\section{A numerical example}

In this section we present a simple numerical example in order to illustrate the effects of a relative audit rule. Suppose that the underlying market structure is a symmetric Cournot duopoly with linear inverse demand (with parameters $a$ and $b$ ) and constant marginal cost $c$. Then the gross profit of firm $i$ is simply given by:

$$
\pi_{i}^{g}\left(q_{i}, q_{j}\right)=: q_{i}\left(a-b\left(q_{i}+q_{j}\right)-c\right) .
$$

As simple relative audit rule is

$$
\beta_{i}\left(d_{i}, d_{j}\right)=:\left\{\begin{array}{ccc}
\rho+x\left(d_{j}-d_{i}\right) & \text { if } & (\rho-1) / x-d_{j} \leq d_{i} \leq \rho / x-d_{j} \\
1 & \text { if } & d_{i}<(\rho-1) / x \\
0 & \text { if } & d_{i}>\rho / x-d_{j}
\end{array}\right.
$$

\footnotetext{
${ }^{11}$ If the game is still supermodular under a relative audit rule then introducing a relative audit rule leads to the prices converging to an equilibrium with lower prices regardless of the Bertrand equilibrium we start at. This follows from Vives (2005), result 5, p 450.
} 


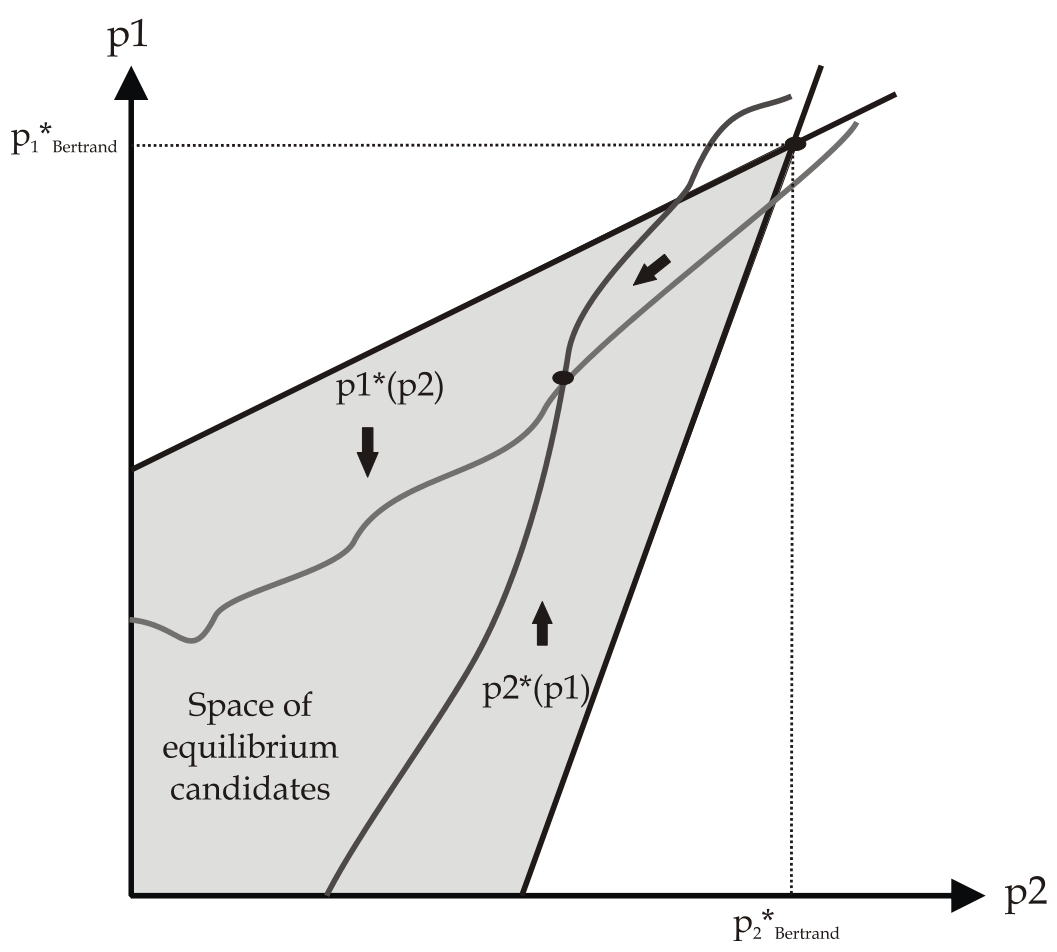

Figure 2: Bertrand equilibrium under relative auditing

We can interpret $\rho$ as the default probability, while $x$ determines the responsiveness of the audit rule to unequal declarations. Furthermore, we assume quadratic evasion costs with a scaling factor $k$ :

$$
C\left(d_{i}, \Pi_{i}^{g}\right):=\frac{\left(\Pi_{i}^{g}-d_{i}\right)^{2}}{k} .
$$

The tax and fine systems are linear with proportionality factors $t$ and $f$. Table 1 shows the impact of a relative audit rule for the parameter vector $(a=10, b=1, c=1, \pi=.2, x=.2, k=20, f=.4, t=.4)$.

In our example the relative audit rule slightly increases the surplus (by 1 percent), while the first dividend - the reduction in tax evasion - is much more pronounced. We conducted a wide range of simulations to get a feel for the size of the different effects. We found that the efficiency gains are usually 


\begin{tabular}{lcccccc}
\hline \hline & $q_{1}+q_{2}$ & $p$ & Surplus & $\Pi_{1,2}^{g}$ & $d_{1,2}$ & evasion $\%$ \\
\hline Relative rule & 6.25 & 3.75 & 36.72 & 8.59 & 7.26 & 15.5 \\
Fixed rule & 6.00 & 4.00 & 36.00 & 9.00 & 6.6 & 26.67 \\
\hline \hline
\end{tabular}

Table 1: Impact of a relative rule

small compared to the reduction in evasion. However, a relative audit rule that does the job of reducing tax evasion and additionally comes with a second dividend - a moderate increase in efficiency - is highly desirable. It has to be noted though that a relative audit rule does not seem suitable as a primary instrument to solve inefficiency problems due to market power.

\section{Conclusions}

This paper has focused on a relatively neglected aspect of tax compliance. It has shown that market structure matters in tax enforcement, a result that is in sharp contrast to the neutrality results that are typical in the literature. We have seen that, in standard models of industrial organisation, the enforcement policy affects firms' behaviour in two dimensions - their market behaviour as well as their compliance behaviour. Appropriate design of the enforcement policy can thus have a "double dividend."

The relative audit rule has an advantage over the independent rule and even over a system without taxation if the efficiency of outputs is concerned. A relative audit rule creates externalities on the declaration of profits, which spill over to the quantity or price decision. In the quantity-competition model this audit rule leads to higher outputs than in a pure Cournot oligopoly. Because an audit regime which treats each firm independently does not impose those externalities, under such a regime the quantity choice is not influenced by the tax-evasion decision and the Cournot quantities are produced. Similar conclusions apply in a price-competition model.

\section{References}

Allingham, M. and A. Sandmo (1972). Income tax evasion: a theoretical analysis. Journal of Public Economics 1, 323-338.

Cowell, F. A. (2004). Carrots and sticks in enforcement. In H. J. Aaron and J. Slemrod (Eds.), The Crisis in Tax Administration, pp. 230-275. Washington DC: The Brookings Instiution. 
Fudenberg, D. and J. Tirole (1991). Game Theory. Cambridge, Mass.: MIT Press.

Lee, K. (1998). Tax evasion, monopoly and nonneutral profit taxes. $\mathrm{Na}$ tional Tax Journal 51, 333-338.

Vives, X. (1999). Oligopoly Pricing: Old Ideas and New Tools. Cambridge, Massachusetts: MIT Press.

Vives, X. (2005). Complementarities and games: New developments. Journal of Economic Literature XLIII, 437-479. 